\title{
Análisis de la evaluación de competencias y su aplicación en un Sistema de Gestión del Aprendizaje. Un caso de studio.
}

\section{Analysis of the Assessment of skills and their application into a Learning Management System. A case study.}

\author{
Cinhtia Maribel González-Segura \\ Universidad Autónoma de Yucatán. Yucatán, México \\ gsegura@correo.uady.mx \\ Michel García-García \\ Universidad Autónoma de Yucatán. Yucatán, México \\ michel.garcia@correo.uady.mx \\ Victor Hugo Menéndez-Domínguez \\ Universidad Autónoma de Yucatán. Yucatán, México \\ mdoming@correo.uady.mx
}

\begin{abstract}
Resumen
Internacionalmente, el enfoque basado en competencias ha sido adoptado en todos los niveles educativos buscando mejorar la calidad de la educación. Sin embargo, aún existen lagunas en el proceso, especialmente en la evaluación, pues no se ha logrado el cambio metodológico necesario para implementarla adecuadamente. El objetivo de este trabajo es analizar cómo se realiza la evaluación de competencias en una universidad, partiendo de la noción de competencia y de las herramientas de un Sistema de Gestión del Aprendizaje que han sido utilizadas exitosamente para realizar dicho proceso. Posteriormente, se describen los resultados preliminares de un diagnóstico aplicado a profesores de la Universidad Autónoma de Yucatán. Los resultados corroboran que son pocas las herramientas que se utilizan con mayor frecuencia y que existe un desconocimiento general acerca de los beneficios potenciales que éstas tienen para la evaluación de competencias genéricas. Finalmente se proponen algunas soluciones que apoyen al docente universitario durante la realización de su función evaluadora bajo el enfoque por competencias.
\end{abstract}

\section{Palabras clave}

Educación superior, Evaluación de competencias, Modelo educativo basado en competencias, Sistema de Gestión del Aprendizaje.

\footnotetext{
Abstract

Internationally, the competence-based approach has been adopted at all levels of education seeking to improve the quality of education. However, there are still gaps in the process, especially in evaluation, since the methodological change necessary to implement it properly has not been achieved. The goal of this paper is to find out how the competency evaluation in a university is carried out, starting from the notion of competence and the tools of a Learning Management System that have been successfully used to carry out this process. Subsequently, we describe the preliminary results of a diagnosis applied to teachers of the Autonomous University of Yucatan. The results corroborate that there are few tools that are used more frequently and that there is a general lack of knowledge about the potential benefits that these have for the evaluation of generic competences. Finally, some solutions are proposed for assist to the university teacher during the performance of his / her evaluation function under the competencies approach.

Key words

Higher Education, Competences Assessment, Learning Management System, CompetencyBased Education Model.
} 


\section{Introducción}

En el siglo XXI, la tendencia internacional en materia educativa ha sido impulsar el Modelo Educativo Basado en Competencias (MEBC) en todos los niveles. Así, se pretende que los estudiantes movilicen sus conocimientos, habilidades, actitudes y valores, demostrando lo que saben hacer con los conocimientos que tienen, al enfrentarse a problemas de diferentes contextos. (Frade, 2012).

En el MEBC, la evaluación se considera un eje fundamental del proceso de formación, debido al gran impacto y potencial que ésta tiene sobre el proceso de aprendizaje del estudiante (Cardona, Velez, y Tobón, 2015), además de que es uno de los procesos ineludibles para analizar lo que ocurre durante el proceso de aprendizaje. Sin embargo, la evaluación es también uno de los procesos que presenta mayores imprecisiones (González-Segura, Menéndez-Domínguez, Sánchez-Arias, y Chan Nuñez, 2014), ya que evaluar competencias requiere enfocarse en la acción y valorar el desempeño del estudiante durante el proceso de la resolución de situaciones específicas en diversos contextos (Valverde Berrocoso, Revuelta Domínguez, y Fernández Sánchez, 2012; Zabala y Arnau, 2007), lo cual es difícil de realizar utilizando instrumentos tradicionales tales como pruebas y exámenes.

A pesar de los múltiples esfuerzos que se han realizado en diversos ámbitos, se observa que aún existen lagunas en el proceso evaluativo por competencias, pues no se ha logrado el cambio metodológico necesario para implementarlo adecuadamente, tal como sugiere Zapata (2010). En algunos casos, se han desarrollado sistemas personalizados para realizar la evaluación de competencias de acuerdo con los requerimientos particulares de las instituciones, pero la frecuencia de uso de estas herramientas parece ser desalentadora. En otros, las herramientas disponibles aún no han sido integradas por completo en las asignaturas (López, Romero, \& Ropero, 2010).

Por otro lado, la tecnología ha sido ampliamente usada en el MEBC pues existen herramientas digitales que permitan la gestión de evidencias de desempeño, permitiendo al docente observar el proceso de aprendizaje de sus estudiantes. Particularmente en la modalidad mixta (b-learning), donde los elementos presenciales se conjugan con los virtuales, los sistemas de gestión del aprendizaje (SGA) ofrecen diversas herramientas que podrían ser aprovechadas para guiar el aprendizaje y complementar los recursos empleados en el salón de clases.

Uno de los Sistemas de gestión del Aprendizaje (SGA) más usados a nivel mundial es Moodle (Modular Object oriented Dynamic Learning Environment), una plataforma de código abierto y modular, ventajas que lo hacen flexible y popular. De acuerdo con (Urbina Nájara, Medina Nieto, y Vargas, 2013) en el 2006 el 7.2\% de las universidades consideraban a Moodle como el sistema de gestión de aprendizaje estándar. En 2007 el porcentaje aumentó a 10.3\% y en 2010 alcanzó el 16.4\% (Green, 2010). La estimación actual de usuarios es de más de 117 millones (Moodle, 2017).

Entre los puntos débiles de Moodle está que los profesores desconocen la efectividad que tienen las herramientas que ofrece para ser usadas en diferentes escenarios pedagógicos (Urbina Nájara et al., 2013). Esto ocasiona que en muchos casos, esta y otras plataformas similares sean usadas meramente como repositorios de documentos (López et al., 2010; Sanchez y Martínez, 2010). Es poco frecuente que los exámenes y

Análisis de la evaluación de competencias y su aplicación en un Sistema de Gestión del Aprendizaje. Cinhtia M. González-Segura, Michel García-García y Victor H. Menéndez-Domínguez. Página 2 de 20 
la retroalimentación se realicen desde ahí (Iglesias, Olmos, Torrecilla y Mena, 2014), particularmente en el enfoque basado en competencias.

En esta investigación se analiza cómo es realizada la evaluación de competencias en una universidad, y cómo son empleadas para este fin las herramientas disponibles en un SGA como Moodle, desde la perspectiva de los docentes universitarios. Esta percepción de los docentes se contrasta con las bondades y finalidades que tienen dichas herramientas, de acuerdo con Seitzinger (2010).

\section{Evaluación de competencias}

Diversos autores, organizaciones e instituciones educativas (De la Orden Oz, 2011; Observatorio de innovación educativa, 2016; Tobón, Pimienta, \& García, 2010; UADY, 2012), han propuesto aproximaciones al concepto de competencia. A partir de lo anterior, en este trabajo se define una competencia como una movilización interrelacionada de un conjunto de conocimientos, habilidades, actitudes y valores que el individuo manifiesta cuando actúa de manera eficaz frente a diversas situaciones problemáticas, en una diversidad de contextos cambiantes; lo cual ocurre en forma cíclica y progresiva, siguiendo la trayectoria de una espiral donde las competencias se incrementan progresivamente en cada ciclo, a partir de las características y experiencias propias de cada sujeto.

Para decidir si un estudiante ha desarrollado cierta competencia, es necesario evaluar su desempeño, idealmente cuando éste se enfrenta a una problemática en la que se requiera movilizar dicha competencia. Sin embargo, esta valoración resulta poco factible por la disponibilidad de espacios y tiempos que permitan vivenciar situaciones reales de aprendizaje, además de la cantidad de alumnos y tiempo disponible de los docentes para realizar su función evaluadora.

Por lo tanto, para realizar una evaluación de competencias centrándose en el proceso, resulta útil diseñar y/o aplicar estrategias didácticas innovadoras que permitan monitorear el proceso que sigue cada individuo a lo largo de su aprendizaje, lo cual puede hacerse de una manera más sistemática y controlada si se aprovechan las ventajas que ofrecen actualmente los recursos informáticos y la tecnología, particularmente los SGA como Moodle.

Sancho Vinuesa y Escudero Viladoms (2012) describen su experiencia al implementar una metodología basada en la evaluación formativa, en la cual los estudiantes son retroalimentados automáticamente al finalizar las actividades incluidas en un curso básico de matemáticas para estudiantes de ingeniería, creado en la plataforma Moodle. La retroalimentación inmediata incluye comentarios y sugerencias sobre el posible origen del error cometido, en su caso. Las autoras afirman que con esta estrategia se ha reducido notablemente el número de estudiantes que abandonan la asignatura, pues el estudiante tiene la oportunidad de regular su propio proceso de aprendizaje y el profesor puede detectar problemáticas a tiempo.

También existen módulos complementarios desarrollados para la plataforma Moodle, como el presentado por Palacios y Espinosa (2012), que permite monitorear el avance individual de las competencias que logran los alumnos de un curso, o el desarrollado

Análisis de la evaluación de competencias y su aplicación en un Sistema de Gestión del Aprendizaje. Cinhtia M. González-Segura, Michel García-García y Victor H. Menéndez-Domínguez. Página 3 de 20 
por Ibarra et al. (2010), denominado EvalCOMIX, que permite realizar la evaluación de competencias de forma semi automatizada. Estos autores afirman que Moodle posee las herramientas básicas para implementar el enfoque por competencias, a las que se ha añadido elementos como la gestión de competencias y sub-competencias con las actividades y ejercicios de un curso, la retroalimentación y los ejercicios de refuerzo, así como la evaluación y coevaluación.

Sin embargo, no se ha encontrado algún trabajo en el que se contrasten las bondades que tienen las herramientas disponibles en la plataforma Moodle con los usos reales que los docentes universitarios hacen de dichas herramientas. En este trabajo, se analiza el caso particular de una universidad pública del sureste mexicano que actualmente se rige por un modelo educativo definido a partir de los principios del MEBC.

\subsection{Evaluación de competencias en la Universidad Autónoma de Yucatán}

La Universidad Autónoma de Yucatán (UADY) es una importante institución pública del sureste mexicano que actualmente se rige por el Modelo Educativo para la Formación Integral (MEFI), el cual se centra en la formación integral del estudiantado por medio de la articulación de seis ejes, ilustrados en la figura 1.

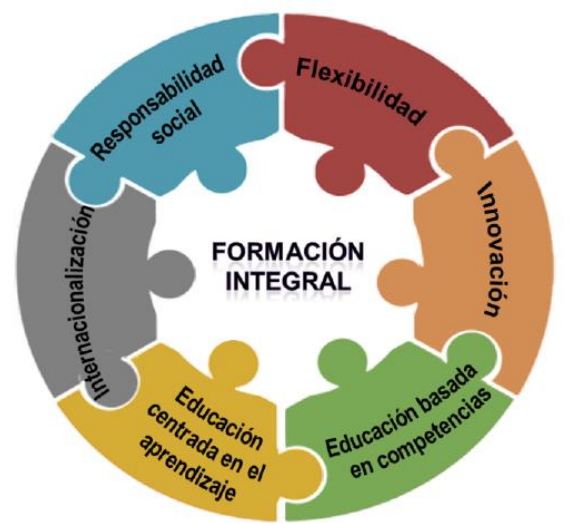

Figura 1. Ejes del modelo educativo de la UADY. Fuente: (UADY, 2012).

Este modelo educativo establece las bases para considerar el currículo como un ámbito de prácticas, relaciones e interacciones en el que todos los actores contribuyan a la formación integral de los estudiantes, con el compromiso de formarlos para incorporarse en la sociedad con una actitud emprendedora y responsable en los ámbitos social, profesional y personal. (UADY, 2012).

Una de las primeras estrategias diseñadas para implementar este modelo educativo consiste en implementar el Programa Institucional de Habilitación en el MEFI (UADY, 2013), que consta de cinco módulos orientados a la capacitación de los académicos para poner en práctica el modelo educativo. Este programa contiene información que va desde la descripción general del modelo hasta la mediación del aprendizaje a través de entornos. Además, recientemente se ha establecido una iniciativa para continuar con la transición hacia el MEFI y que forma parte del Plan de Desarrollo Institucional 20142022, se trata del Programa Institucional de Actualización Docente (PIAD), orientado a la habilitación del personal académico en el desarrollo de las competencias del perfil docente de la UADY, acorde con el perfil propuesto por Rangel (2015). Este programa se conforma por nueve diplomados y seis áreas de habilitación: estrategias de enseñanza y aprendizaje, evaluación educativa, gestión de la información y el conocimiento,

Análisis de la evaluación de competencias y su aplicación en un Sistema de Gestión del Aprendizaje. Cinhtia M. González-Segura, Michel García-García y Victor H. Menéndez-Domínguez. Página 4 de 20 
entornos virtuales de aprendizaje, inglés como segunda lengua e inglés para propósitos académicos y científicos (UADY, 2017).

Por otra parte, la plataforma Moodle es el SGA que se ha adoptado en la UADY desde el año 2004 para atender las necesidades de la educación en línea (Esperón-Hernández \& Ley Fuentes, 2005). Desde entonces, esta plataforma se ha empleado para gestionar los cursos que se imparten hasta la fecha (Reyes Cabrera, Guillermo y Guillermo, Quiñones Pech, y Zapata González, 2013), los cuales están orientados a una formación integral del estudiante y a la enseñanza-aprendizaje por competencias. Sin embargo, a partir del 2012 Moodle se ha convertido en la plataforma institucional para implementar el MEFI. De igual manera, la UADY cuenta con repositorios de recursos de educativos que pueden ser integrados a Moodle, como es el caso de la plataforma AGORA (Ayuda para la Gestión de Objetos Reusables de Aprendizaje), que propone un marco de trabajo donde las actividades y tareas asociadas a la gestión de Objetos de Aprendizaje se intercomuniquen para facilitar su control y ejecución (Menéndez-Domínguez, Castellanos-Bolaños, Cambranes Martínez, Medina Peralta, y Zapata González, 2013).

\subsection{Herramientas de Moodle}

Las herramientas que contiene Moodle presentan leves diferencias entre versiones, principalmente en el nombre de las actividades, aunque básicamente la funcionalidad es la misma. En la Tabla 1 se muestran las herramientas disponibles en la versión 3.1.

Tabla 1. Herramientas disponibles en Moodle 3.1.

\begin{tabular}{|c|c|}
\hline Herramienta & Descripción \\
\hline \multicolumn{2}{|r|}{ Actividades } \\
\hline Asistencia & $\begin{array}{l}\text { Permite a los profesores mantener un registro en línea de las } \\
\text { asistencias, con diferentes eventos o sesiones en cada curso. }\end{array}$ \\
\hline Base de datos & $\begin{array}{l}\text { Permite al maestro o al estudiante, mostrar y buscar datos acerca de } \\
\text { cualquier tópico. El formato permite manejar imágenes, archivos, } \\
\text { URLs, números y texto, entre otros. }\end{array}$ \\
\hline Chat & $\begin{array}{l}\text { Permite que los participantes tengan una discusión sincrónica, } \\
\text { basada en texto, en tiempo real en la web. }\end{array}$ \\
\hline $\begin{array}{l}\text { Elección } \\
\text { (Consulta) }\end{array}$ & $\begin{array}{l}\text { Permite al profesor hacer una sola pregunta y ofrecer una selección } \\
\text { de respuestas posibles. }\end{array}$ \\
\hline $\begin{array}{l}\text { Encuesta } \\
\text { predefinida }\end{array}$ & $\begin{array}{l}\text { Proporciona varios instrumentos de encuestas verificadas, } \\
\text { incluyendo COLLES (Constructivist On-Line Learning } \\
\text { Environment Survey) y ATTLS (Attitudes to Thinking and Learning } \\
\text { Survey), que han sido útiles en la evaluación y estimulación del } \\
\text { aprendizaje en ambientes en línea. }\end{array}$ \\
\hline Examen & $\begin{array}{l}\text { Permite al profesor crear exámenes con preguntas de varios tipos: } \\
\text { opción múltiple, falso/verdadero, relacionar columnas, respuesta } \\
\text { corta y calculada. Estas preguntas se almacenan en un banco de } \\
\text { preguntas y pueden ser reutilizadas. }\end{array}$ \\
\hline Foro & $\begin{array}{l}\text { Permite a los participantes tener discusiones asincrónicas, que } \\
\text { suceden a lo largo de un largo período de tiempo. }\end{array}$ \\
\hline Glosario & $\begin{array}{l}\text { Permite a los participantes crear y mantener una lista de } \\
\text { definiciones, como en un diccionario, o colectar y organizar } \\
\text { recursos o información. }\end{array}$ \\
\hline
\end{tabular}

Análisis de la evaluación de competencias y su aplicación en un Sistema de Gestión del Aprendizaje. Cinhtia M. González-Segura, Michel García-García y Victor H. Menéndez-Domínguez. Página 5 de 20 


\begin{tabular}{|c|c|}
\hline $\begin{array}{l}\text { Herramienta } \\
\text { externa }\end{array}$ & $\begin{array}{l}\text { Permite a los estudiantes interactuar con recursos educativos y } \\
\text { actividades alojadas en otros sitios de internet. }\end{array}$ \\
\hline Lección & $\begin{array}{l}\text { Permite al profesor proporcionar contenidos y/o actividades } \\
\text { presentadas de tal forma que un conjunto lineal de páginas de } \\
\text { contenidos o actividades instruccionales pueden ofrecer una } \\
\text { variedad de caminos o de opciones para el estudiante. }\end{array}$ \\
\hline Paquete SCORM & $\begin{array}{l}\text { Colección de archivos que están empaquetados de acuerdo con el } \\
\text { estándar acordado para los objetos de aprendizaje. }\end{array}$ \\
\hline $\begin{array}{l}\text { Retroalimentación } \\
\text { (Módulo de } \\
\text { encuesta) }\end{array}$ & $\begin{array}{l}\text { Permite al profesor crear encuestas personalizadas para colectar } \\
\text { retroalimentación de los participantes, con diferentes tipos de } \\
\text { preguntas como: opción múltiple, falso/verdadero, o respuesta de } \\
\text { texto. Se pueden crear preguntas sin calificación. }\end{array}$ \\
\hline Taller & $\begin{array}{l}\text { Actividad para el trabajo en grupo mediante la evaluación por } \\
\text { pares, con un gran número de opciones. Permite a los estudiantes } \\
\text { enviar sus trabajos a través de una herramienta de texto online y } \\
\text { archivos adjuntos, así como la revisión y evaluación por pares del } \\
\text { trabajo de los estudiantes. }\end{array}$ \\
\hline Tarea & $\begin{array}{l}\text { Permite al profesor comunicar quehaceres, recolectar los trabajos y } \\
\text { proporcionar calificaciones y retroalimentación. Los estudiantes } \\
\text { pueden enviar sus trabajos para que los profesores los califiquen y } \\
\text { proporcionen retroalimentación. }\end{array}$ \\
\hline Wiki & $\begin{array}{l}\text { Permite a los participantes añadir y editar una colección de páginas } \\
\text { web. Un wiki puede ser colaborativo, donde todos pueden editarlo, } \\
\text { o puede ser individual. }\end{array}$ \\
\hline \multicolumn{2}{|r|}{ Recursos } \\
\hline Archivo & $\begin{array}{l}\text { Permite al profesor subir un archivo como recurso para un curso. El } \\
\text { archivo se puede mostrar dentro de la interfaz del curso, o bien, } \\
\text { hacer que los estudiantes descarguen el archivo. }\end{array}$ \\
\hline Carpeta & $\begin{array}{l}\text { Permite al profesor mostrar un número de archivos relacionados } \\
\text { que están dentro de una sola carpeta. Puede subirse un archivo } \\
\text { comprimido en ZIP y luego descomprimirse para ser mostrado, o se } \\
\text { puede crear una carpeta vacía y subir archivos dentro de ella. }\end{array}$ \\
\hline Etiqueta & $\begin{array}{l}\text { Permite al profesor insertar texto, elementos multimedia e } \\
\text { hipervínculos en una página del curso. Las etiquetas son muy } \\
\text { versátiles y pueden ayudar a mejorar la apariencia de un curso. }\end{array}$ \\
\hline Libro & $\begin{array}{l}\text { Permite al profesor crear un recurso multi-página en un formato } \\
\text { similar a un libro, con capítulos y subcapítulos. Los libros pueden } \\
\text { contener archivos multimedia y texto, son útiles para mostrar } \\
\text { pasajes largos de información que puede dividirse en secciones. }\end{array}$ \\
\hline Página & $\begin{array}{l}\text { Permite al profesor crear una página web empleando el editor de } \\
\text { texto. En una página se pueden mostrar texto, imágenes, sonido, } \\
\text { video, enlaces de internet y código incrustado }\end{array}$ \\
\hline $\begin{array}{l}\text { Paquete contenido } \\
\text { IMS }\end{array}$ & $\begin{array}{l}\text { Es una colección de archivos empaquetados de acuerdo con un } \\
\text { estándar acordado, de tal forma que puede ser reutilizado en } \\
\text { diferentes plataformas. Este módulo permite subir un paquete de } \\
\text { contenidos como un archivo ZIP y añadirlo como un recurso. }\end{array}$ \\
\hline URL & $\begin{array}{l}\text { Permite al profesor proporcionar un enlace de internet para un } \\
\text { recurso del curso, con varias opciones de visualización. }\end{array}$ \\
\hline
\end{tabular}

Análisis de la evaluación de competencias y su aplicación en un Sistema de Gestión del Aprendizaje. Cinhtia M. González-Segura, Michel García-García y Victor H. Menéndez-Domínguez. Página 6 de 20 
Fuente: Elaboración propia a partir de (Moodle, 2017).

En la tabla 2, se muestran las finalidades pedagógicas asociadas a cada herramienta, de acuerdo con Seitzinger (2010). Las columnas marcadas con XX indican que esa finalidad es la más recomendada y la $\mathrm{X}$ indica que es apropiada en menor grado. Las celdas vacías indican que no se ha establecido relación entre la herramienta y la finalidad pedagógica correspondiente.

Tabla 2. Finalidades pedagógicas de las herramientas de Moodle.

\begin{tabular}{|c|c|c|c|c|}
\hline Herramienta & $\begin{array}{c}\text { Transferencia } \\
\text { de } \\
\text { información }\end{array}$ & $\begin{array}{l}\text { Comunicación } \\
\text { e interacción }\end{array}$ & $\begin{array}{c}\text { Elaboración } \\
\text { colaborativa } \\
\text { de contenidos }\end{array}$ & $\begin{array}{c}\text { Evaluación } \\
\text { del } \\
\text { aprendizaje }\end{array}$ \\
\hline \multicolumn{5}{|l|}{ Asistencia } \\
\hline Base de datos & $X$ & $X$ & XX & $X$ \\
\hline Chat & $\mathrm{X}$ & $\mathrm{XX}$ & $\mathrm{XX}$ & $\mathrm{X}$ \\
\hline Elección (Consulta) & & & & $\mathrm{X}$ \\
\hline Encuesta predefinida & & & & $\mathrm{X}$ \\
\hline Examen & & & & $\mathrm{XX}$ \\
\hline Foro & $X$ & $\mathrm{XX}$ & $X X$ & $\mathrm{X}$ \\
\hline Glosario & $\mathrm{XX}$ & $\mathrm{X}$ & $\mathrm{XX}$ & $X$ \\
\hline Herramienta externa & $\mathrm{X}$ & $\mathrm{X}$ & $\mathrm{X}$ & $\mathrm{X}$ \\
\hline Lección & $\mathrm{XX}$ & & & $\mathrm{XX}$ \\
\hline Paquete SCORM & $\mathrm{XX}$ & & & $\mathrm{XX}$ \\
\hline $\begin{array}{l}\text { Retroalimentación } \\
\text { (mod. de encuesta) }\end{array}$ & & XX & & \\
\hline Taller & & & $X$ & $\mathrm{XX}$ \\
\hline Tarea & & & & XX \\
\hline Wiki & $\mathrm{XX}$ & $\mathrm{X}$ & $\mathrm{XX}$ & $\mathrm{X}$ \\
\hline Archivo & $\mathrm{XX}$ & & $\mathrm{X}$ & $\mathrm{X}$ \\
\hline Carpeta & $\mathrm{XX}$ & & $X$ & $\mathrm{X}$ \\
\hline \multicolumn{5}{|l|}{ Etiqueta } \\
\hline Libro & $\mathrm{XX}$ & $\mathrm{X}$ & $X$ & $\mathrm{X}$ \\
\hline Página & $\mathrm{XX}$ & $X$ & $\mathrm{X}$ & $X$ \\
\hline Paq. contenido IMS & $\mathrm{XX}$ & & & \\
\hline URL & $\mathrm{XX}$ & $\mathrm{X}$ & $\mathrm{X}$ & \\
\hline
\end{tabular}

Fuente: Adaptación propia a partir de (Moodle, 2017; Seitzinger, 2010)

Además de las herramientas anteriores, existen otras disponibles en la Web 2.0 que se pueden integrar en la plataforma Moodle, por ejemplo: Blogger, Wiki, Slideshare, slideboom, Sliderocket, Issu, Scribd, Youblisher, YouTube, Flickr, Audacity. Otras herramientas de autor que se pueden utilizar para desarrollar contenidos e incorporarlos posteriormente son: Prezi, CmapTools, FreeMind, Jclic, Ardora, Edilim, HotPotatoes, Malted, Educaplay, Cuadernia, eXelearning, iSpring, iSpring Quiz Maker, My Udutu, Audioboo, Ivoox, entre otros. (Jiménez, 2013). 


\section{Metodología}

Se implementa un estudio de caso por considerar pertinente hacerlo cuando la investigación se ocupa tanto de una cuestión descriptiva (qué pasa) como de una pregunta explicativa (cómo o por qué sucede algo), (Yin, 2003). Además, si se considera que el estudio de caso pretende describir y explicar un fenómeno a partir de la comprensión del problema y usando un caso particular como una ilustración específica de un contexto delimitado (Creswell, 2007), esta metodología resulta apropiada para analizar el caso de una universidad en la que se realiza el análisis descrito.

Aquí, el contexto está delimitado por un entorno constituido por una dependencia educativa, la fuente de información es la encuesta respondida por los profesores universitarios, a través de un instrumento digital. De esta manera, se busca responder las preguntas de investigación planteadas y avanzar hacia la comprensión de la situación que se analiza: la evaluación de competencias a partir de un conjunto de herramientas disponibles en la plataforma Moodle.

\subsection{Contexto y objetivos de la investigación}

El objetivo de esta investigación es analizar cuáles herramientas utiliza el docente universitario para llevar a cabo su función evaluadora bajo el MEBC en un ambiente mixto o b-learning, con qué fines y con qué frecuencia usa las herramientas disponibles en un SGA, para posteriormente contrastar si hay coincidencia entre la percepción docente y las funciones pedagógicas asociadas a cada herramienta.

Las preguntas de investigación que se busca responder son: ¿Cuáles son las herramientas comúnmente usadas por los profesores, al realizar su función evaluadora? ¿Cuáles modalidades de evaluación están implementando los docentes y con qué herramientas lo hacen? Y, ¿las finalidades pedagógicas asociadas a las herramientas de Moodle coinciden con las percepciones que tienen los docentes universitarios, respecto de las mismas?

En el MEFI, la UADY declara 22 competencias genéricas que los egresados deben desarrollar independientemente del programa educativo que cursen (UADY, 2013). Sin embargo, en Moodle las herramientas disponibles permiten realizar actividades pertenecientes a alguna de las siguientes categorías: transferencia de información, comunicación e interacción, elaboración colaborativa de contenidos y evaluación del aprendizaje (Seitzinger, 2010).

Para conocer la perspectiva docente de los profesores de la UADY, se aplicó un estudio de caso dirigido a una población conformada por los profesores de educación superior, quienes fueron invitados a responder una encuesta en línea. En este nivel educativo, la UADY está conformada por 15 facultades agrupadas en 5 campus: "Ciencias de la Salud", "Biológicas y agropecuarias", "Ciencias exactas e ingenierías", "Sociales, económico administrativas y humanidades" y "Arquitectura, arte y diseño". La planta docente de la UADY consta de 777 profesores de tiempo completo y 1,756 profesores en total (UADY, 2014).

Se diseñó una encuesta que fue digitalizada utilizando los formularios de Google y fue distribuida a la comunidad universitaria través de una lista de correo institucional, en la

Análisis de la evaluación de competencias y su aplicación en un Sistema de Gestión del Aprendizaje. Cinhtia M. González-Segura, Michel García-García y Victor H. Menéndez-Domínguez. Página 8 de 20 
que se encuentran los correos electrónicos de todos los docentes. La encuesta aplicada estuvo disponible en línea y fue respondida en el período comprendido del 15 de junio al 28 de agosto de 2015.

\subsection{Instrumento}

Una vez diseñado el instrumento, éste fue validado mediante la técnica denominada juicio de expertos (Gutiérrez-Castillo, Cabero-Almenara, y Estrada-Vidal, 2017), para lo cual la encuesta fue presentada a un grupo de expertos en educación mediada por tecnologías, de diferentes universidades nacionales en el ámbito de nivel superior, quienes valoraron los ítems en cuanto a claridad y pertinencia, sugirieron algunos cambios mínimos y validaron el instrumento.

La encuesta consta de 3 secciones y 9 reactivos en total, todos de opción múltiple con posibilidad de una respuesta abierta en caso necesario. En la primera sección, se incluyeron 5 preguntas cerradas solicitando indicar la disciplina de formación, el grado máximo de estudios, el rango de años como docente universitario, el rango de años que el profesor lleva utilizando una plataforma e-learning como Moodle y el nivel de experiencia que considera que él mismo posee respecto al uso de la misma. Estos datos permitieron conocer la distribución de disciplinas de los profesores participantes y el perfil docente que éstos poseen.

En la segunda sección de la encuesta se incluyeron 4 reactivos diseñados en forma matricial, donde las filas corresponden a las herramientas disponibles en la versión de la plataforma institucional de la UADY y las columnas corresponden, en los dos primeros reactivos, a una escala de Likert con 5 posibles respuestas y, en los dos últimos, corresponden a una lista de opciones, como se muestra en la Figura 2. Las columnas corresponden a las finalidades pedagógicas de Moodle (Seitzinger, 2010), desglosando las formas de evaluación.

\begin{tabular}{|c|c|c|c|c|c|c|c|}
\hline & 1 & 2 & 3 & 4 & 5 & 6 & No sé \\
\hline Archivo & 0 & 0 & 0 & 0 & 0 & 0 & 0 \\
\hline Carpeta & 0 & 0 & 0 & 0 & 0 & 0 & 0 \\
\hline Libro & 0 & 0 & 0 & 0 & 0 & 0 & 0 \\
\hline Página & 0 & 0 & 0 & O & 0 & 0 & 0 \\
\hline URL & 0 & 0 & $\mathrm{O}$ & O & 0 & 0 & 0 \\
\hline Asistencia & 0 & 0 & 0 & 0 & 0 & 0 & 0 \\
\hline Base de datos & 0 & 0 & 0 & 0 & 0 & 0 & 0 \\
\hline Consultas & 0 & 0 & 0 & 0 & $\mathrm{O}$ & 0 & 0 \\
\hline Cuestionario & 0 & O & 0 & 0 & 0 & 0 & 0 \\
\hline Encuesta & 0 & 0 & 0 & 0 & 0 & 0 & 0 \\
\hline Foro & 0 & 0 & 0 & 0 & 0 & 0 & 0 \\
\hline Glosario & 0 & 0 & 0 & $\mathrm{O}$ & 0 & 0 & 0 \\
\hline $\begin{array}{l}\text { Herramienta } \\
\text { externa }\end{array}$ & 0 & 0 & 0 & 0 & 0 & 0 & 0 \\
\hline
\end{tabular}

Figura 2. Extracto del instrumento digital que fue creado utilizando los formularios de google y distribuido a los profesores de la UADY a través de una lista de correo institucional.

En el instrumento aplicado, la evaluación del aprendizaje se desglosó incluyendo la autoevaluación del estudiante y la coevaluación entre estudiantes, con el fin de conocer 
en qué medida se están atendiendo, tal como se indica en el MEBC (Observatorio de innovación educativa, 2016; Palacios Burgos y Espinosa Martín, 2012).

Los datos recabados permitieron conocer con qué frecuencia el profesor usa las herramientas de dicha plataforma, la usabilidad (facilidad/dificultad) que observa en el uso de las mismas, cuáles de las competencias listadas considera que se favorecen más con cada herramienta y cuáles de los objetivos listados considera que se impactan más al usar cada una de ellas.

\subsection{Procedimiento}

En primer lugar, se presentan los datos descriptivos de la muestra de profesores universitarios que decidieron responder voluntariamente la encuesta digital distribuida. Las dimensiones consideradas en el estudio son: disciplina de formación, grado máximo de estudios, antigüedad como docente universitario, antigüedad utilizando una plataforma e-Learning como Moodle y nivel de experiencia en el uso de una plataforma como esta, las cuales se contrastan con la frecuencia de uso, objetivos con que se utiliza y facilidad/dificultad de uso de cada herramienta. A su vez, estas dimensiones son contrastadas con las funciones pedagógicas asociadas a cada herramienta, con el fin de constatar la existencia de diferencias significativas entre la percepción de los profesores y las finalidades pedagógicas asociadas a dichas herramientas.

\subsection{Muestra participante}

Durante el período en que estuvo disponible en línea, la encuesta fue respondida voluntariamente por 255 profesores universitarios, lo cual corresponde a un $92 \%$ de confiabilidad. Las disciplinas de especialidad de los profesores participantes son diversas, en la proporción que se observa en la Gráfica 1.
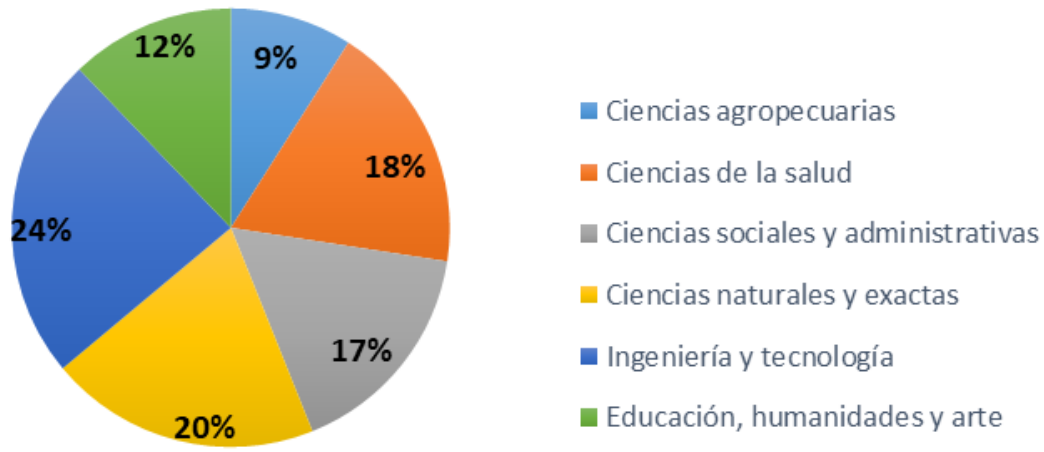

Gráfica 1. Disciplinas a las que pertenece la muestra de profesores participantes.

Para analizar el perfil de los participantes se solicitó indicar, además de su disciplina de formación por áreas, su grado máximo de estudios, su antigüedad como docente universitario, su antigüedad usando una plataforma e-learning como Moodle y su nivel de experiencia en el uso de la plataforma.

En cuanto a la antigüedad como docente universitario se definieron 5 intervalos: menos de 5 años, entre 5 y 10, entre 11 y 15, entre 16 y 20 y más de 20 años. Asimismo, la antigüedad usando una plataforma e-learning se indicó a partir de las opciones: no he usado alguna plataforma, menos de 3 años, entre 3 y 6 años, entre 6 y 9 años, o bien, más de 9 años. 


\section{Resultados}

\subsection{Dimensión perfil del docente universitario}

La descripción de la muestra, considerando las variables que contribuyen a definir el perfil de los docentes universitarios que participaron voluntariamente en el estudio, queda descrito a continuación:

a) Formación académica: Máximo grado académico. el $46 \%$ de los profesores cuenta con doctorado, el $34 \%$ con maestría, el $14 \%$ con licenciatura y el $6 \%$ posee alguna especialización.

b) Tiempo: Años que lleva usando una plataforma como Moodle. El $84 \%$ de los profesores encuestados manifestó que sí utiliza alguna plataforma ELearning, como Moodle. El 43\% indicó que la ha usado durante menos de 3 años, el $31 \%$ entre 3 y 6 años y el $8 \%$ entre 6 y 9 años y nadie manifestó haberla usado por on más de 9 años.

c) Experiencia: Nivel de experiencia en el uso de SGA. El 10\% manifiesta que tiene una experiencia nula, el 20\% incipiente, el $50 \%$ considera que se encuentra en desarrollo, el $17 \%$ considera tener un nivel maduro y el $3 \%$ se considera experto.

\subsection{Dimensión experticia personal en el uso de una plataforma e-learning}

El nivel de experticia personal que cada profesor considera poseer respecto a las herramientas de Moodle es bastante diverso en función de su disciplina de formación y los años que lleva usando la plataforma. En la Gráfica 2 se ilustra esta distribución y se observa que la mayoría de los participantes que considera poseer un nivel en desarrollo lleva 6 años o menos haciendo uso de la plataforma. También se aprecia que existe una correlación directa entre quienes no han usado alguna plataforma y quienes manifiestan tener una experiencia incipiente o nula.

\subsection{Herramientas usadas con mayor frecuencia por los docentes}

Para obtener la frecuencia de uso que tienen las herramientas, se consideró la cantidad de menciones que cada herramienta tuvo y este valor se multiplicó por el factor de frecuencia correspondiente en la escala de Richter del instrumento, siendo muy frecuente $=4$ puntos, bastante $=3$ puntos, intermedio $=2$ puntos, poco $=1$ punto, nulo $=$ 0 puntos. En la Gráfica 3 se muestra cuál es la frecuencia con que los docentes utilizan las herramientas de Moodle. Se observa que las herramientas con mayor frecuencia de uso son: archivo (636 puntos), tarea (578), carpeta (481), foro (380) y URL (378). Asimismo, las herramientas con menos frecuencia de uso son: paquete SCORM (72 puntos), HotPotatoes (84), taller (133) y herramienta externa (176).

\subsection{Usabilidad (facilidad de uso) de las herramientas disponibles.}

La usabilidad de las herramientas fue determinada con base en las respuestas que los profesores indicaron para la pregunta ¿cuál es la facilidad/dificultad de uso que usted observa en las herramientas de Moodle? Los puntajes para cada herramienta se calcularon ponderando la cantidad de menciones que tuvo cada una de las 5 respuestas posibles y posteriormente se multiplicó por un valor asociado a ellas, donde muy fácil de usar se asoció con el valor +2 , fácil de usar se asoció con +1 , intermedia con 0 , difícil de usar con -1, muy difícil de usar con -2.

Análisis de la evaluación de competencias y su aplicación en un Sistema de Gestión del Aprendizaje. Cinhtia M. González-Segura, Michel García-García y Victor H. Menéndez-Domínguez. Página 11 de 20 


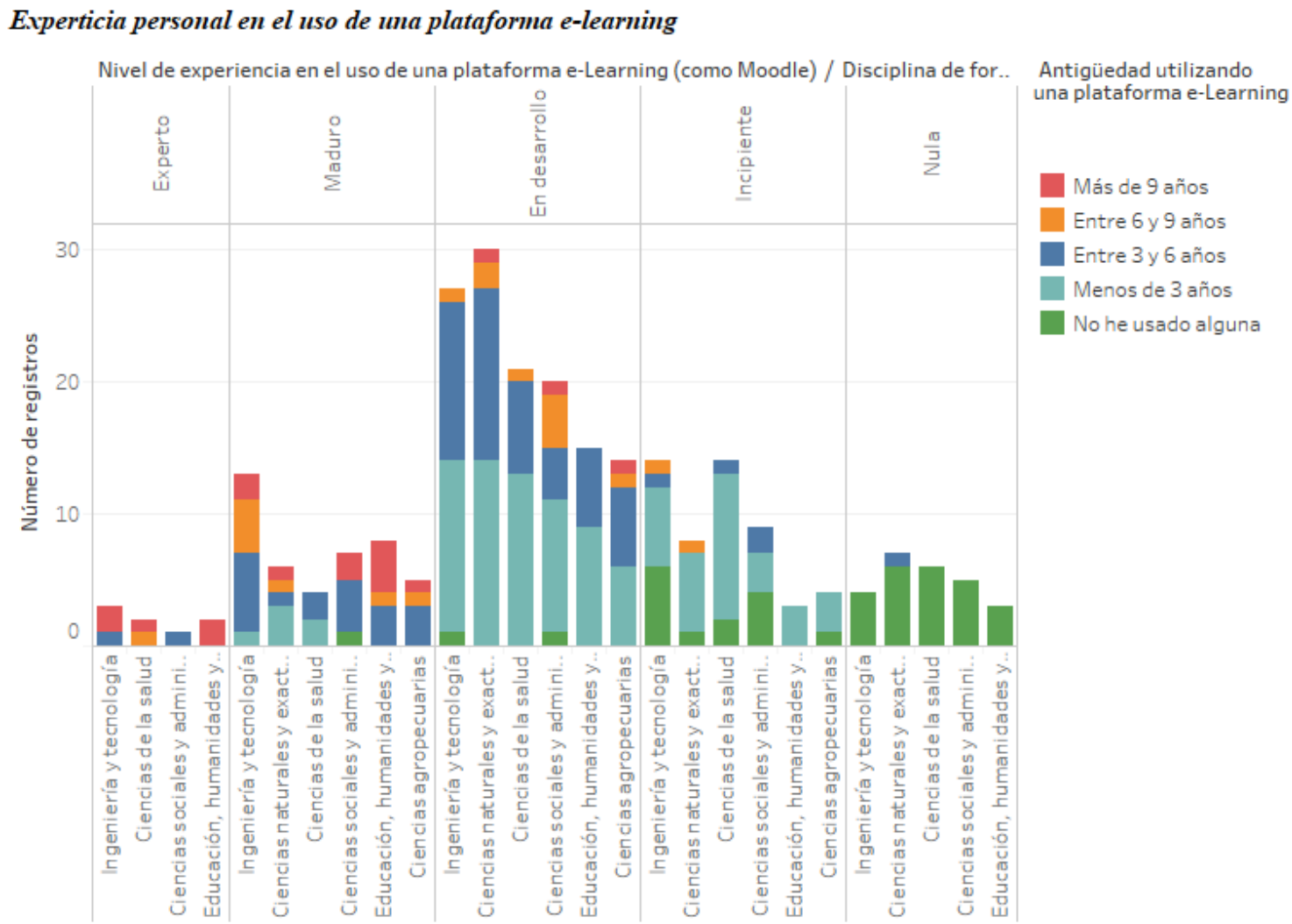

Gráfica 2. Nivel de experticia de los participantes en el uso de una plataforma e-learning como Moodle.

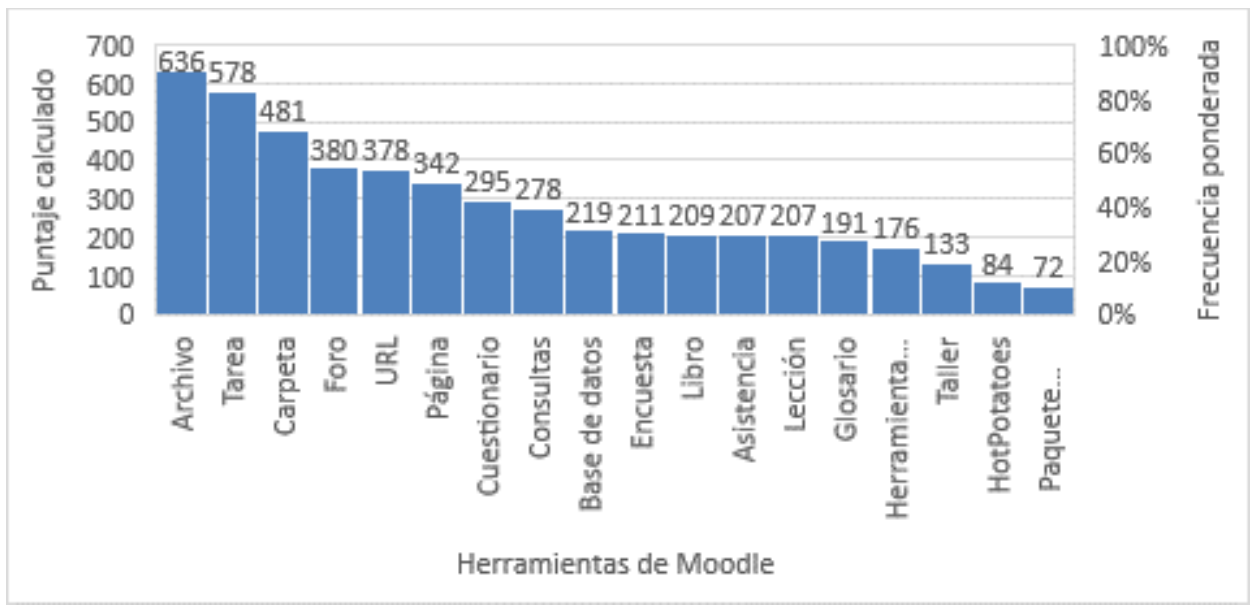

Gráfica 3. Herramientas de Moodle y sus frecuencias de uso ponderadas.

En la Gráfica 3 se muestra cuál es la frecuencia con que los docentes utilizan las herramientas de Moodle. Las herramientas ordenadas de mayor a menos usabilidad (facilidad de uso), con su respectivo puntaje calculado son: archivo (363 puntos), carpeta (312), tarea (268), URL (228), foro (225), página (209), cuestionario (174), libro (173), consultas (154), glosario (144), encuesta (140), asistencia (129), base de datos (101), lección (96), herramienta externa (68), taller (61), HotPotatoes (30) y paquete SCORM (7 puntos). 


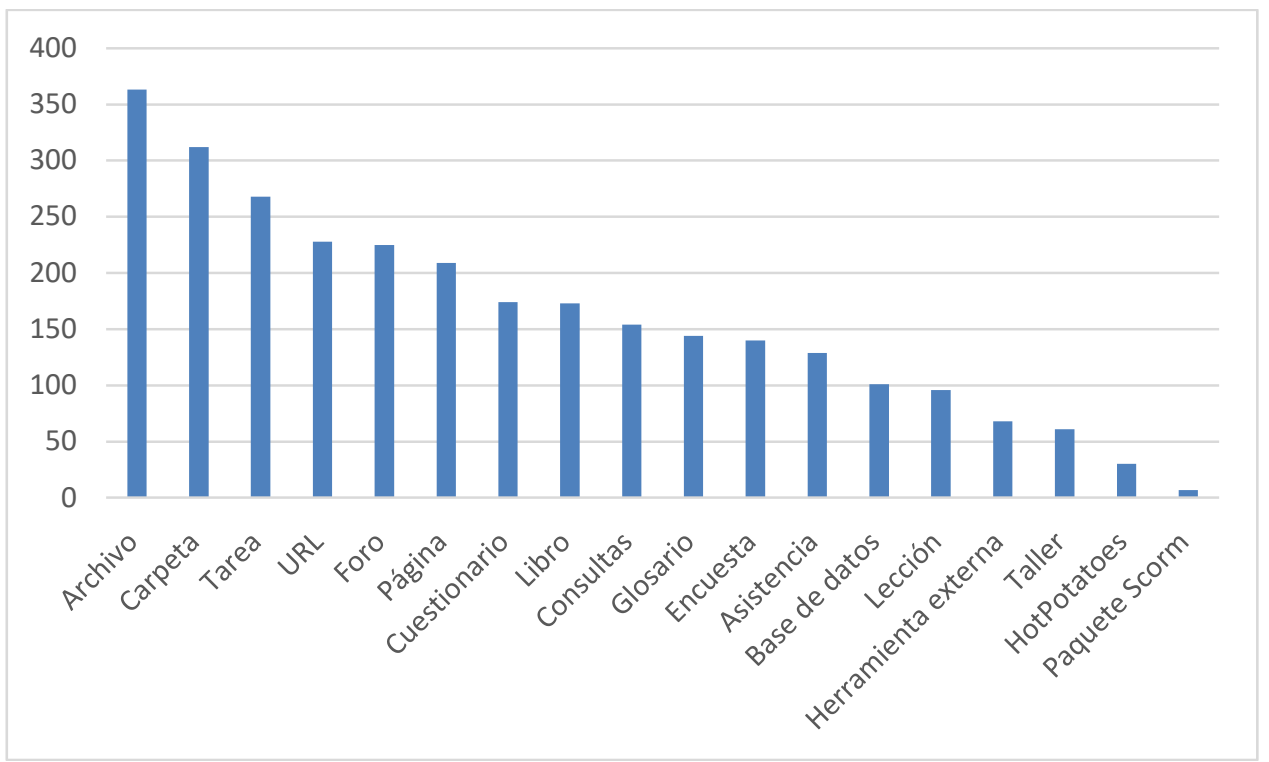

Gráfica 4. Facilidad/dificultad de uso de las herramientas de Moodle.

Cabe señalar que en la Gráfica 3 se ha omitido la cantidad de respuestas no lo he utilizado por considerar que ésta tiene un significado diferente. Los porcentajes de profesores que indicaron esta respuesta para las herramientas, ordenados de mayor a menor son los siguientes: hotPotatoes (52\%), paquete Scorm (50\%), taller (45\%), herramienta externa (43\%), lección (40\%), asistencia (38\%), glosario, base de datos y consultas (36\%), encuesta (34\%), libro (33\%), URL (28\%), cuestionario (27\%), página (26\%), foro (21\%), archivo (14\%), tarea (19) y carpeta (18\%).

\subsection{Objetivos que se consideran más favorecidos.}

En cuanto a los objetivos o funcionalidades pedagógicas que los profesores consideran que son más favorecidas con cada herramienta, se mencionan a continuación las de mayor incidencia. Para realizar la transferencia de la información, el $79 \%$ de los profesores indicaron que usan la herramienta archivo, el 64\% usa la carpeta, el 63\% usa el libro, el 56\% usa la URL, el 52\% la página y en las demás herramientas se observa menos incidencia. Para la comunicación e interacción, el 55\% usa el foro, el $43 \%$ la asistencia, el $38 \%$ las consultas y las demás herramientas en menor grado. Para la elaboración colaborativa de contenidos, la herramienta taller es usada por el $29 \%$ de los profesores, el $24 \%$ usa el glosario, el $20 \%$ usa la base de datos.

Para la evaluación del aprendizaje, el 39\% de los profesores usa la herramienta tarea, el $36 \%$ usa el cuestionario, el $24 \%$ usa la encuesta y $23 \%$ la lección. Para la autoevaluación del aprendizaje, la herramienta con mayor uso es el HotPotatoes con $24 \%$ y el cuestionario con $23 \%$. La coevaluación presenta menos menciones, el $15 \%$ de los profesores usa el foro, tanto la encuesta, como la herramienta externa y el taller son usadas por el $10 \%$ de los profesores, el resto de las herramientas presenta menor ocurrencia. En la Gráfica 5 se muestra lo anterior. 


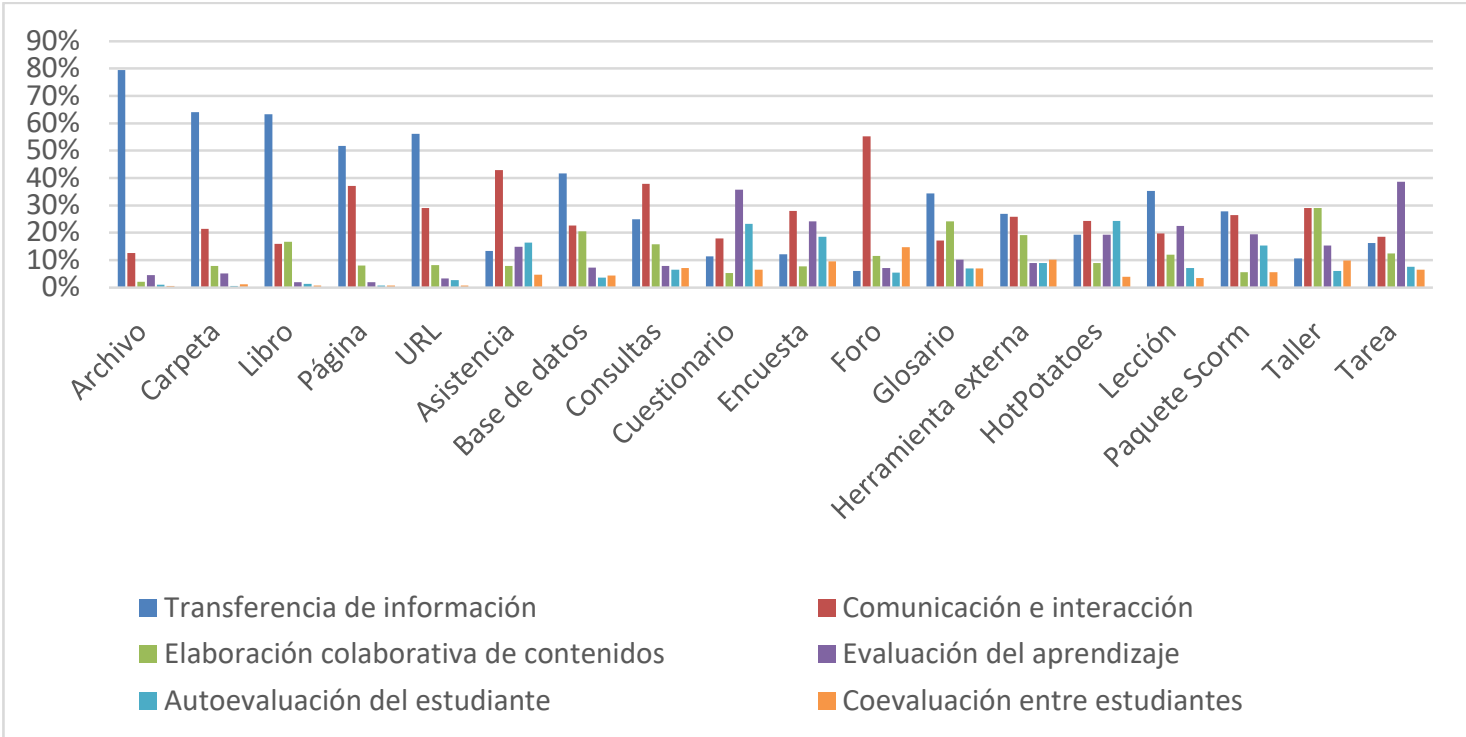

Gráfica 5. Objetivos pedagógicos que los docentes indicaron que son más favorecidos con cada herramienta

\subsection{Competencias que se consideran más favorecidas.}

Ante la pregunta planteada respecto a cuáles son las herramientas de Moodle que más favorecen las seis categorías de competencias genéricas incluidas en el instrumento, se observa que en muchas de las herramientas son altos los porcentajes de profesores que no respondieron (archivo: 27\%, carpeta: 36\%, libro: 49\%, página: 48\%, URL: $51 \%$, asistencia: 46\%, base de datos: 48\%, consultas: 45\%, cuestionario: 39\%, encuesta: 41\%, foro: $27 \%$, glosario: $42 \%$, herramienta externa: $62 \%$, hotpotatoes: $71 \%$, lección: 49\%, paquete scorm: $75 \%$, taller: $52 \%$ y tarea: $32 \%$ ). Una de las competencias que los profesores sí asociaron con varias herramientas que se pueden utilizar para su desarrollo es la responsabilidad, para la que se indicaron 11 herramientas con las que ésta puede promoverse: asistencia, tarea, lección, archivo, carpeta, cuestionario, página, URL, consulta, paquete SCORM y HotPotatoes. Las otras competencias: trabajo en equipo, liderazgo, cooperación, comunicación e iniciativa tuvieron menos asociaciones con las herramientas disponibles. Estas categorías de competencias genéricas fueron incluidas a partir de las funcionalidades pedagógicas establecidas en la guía de herramientas para profesores (Seitzinger, 2010).

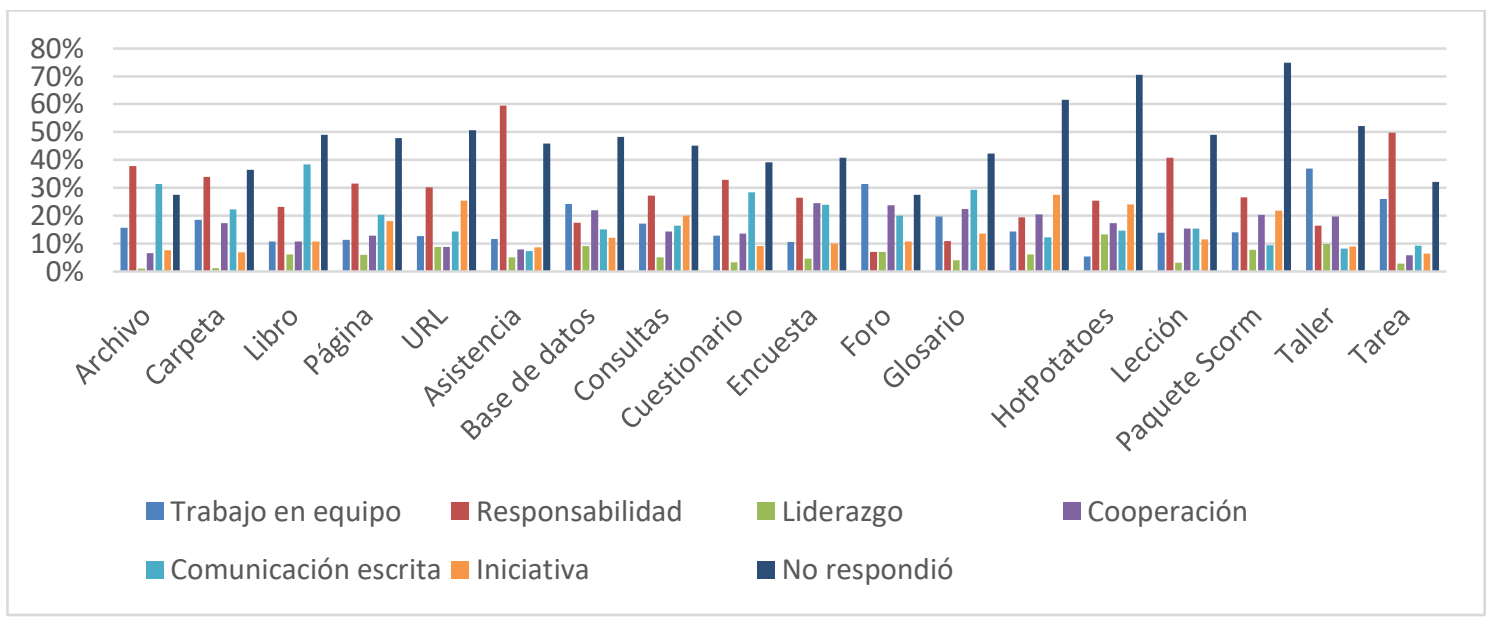

Análisis de la evaluación de competencias y su aplicación en un Sistema de Gestión del Aprendizaje. Cinhtia M. González-Segura, Michel García-García y Victor H. Menéndez-Domínguez. Página 14 de 20 
Gráfica 6. Competencias que los docentes consideran que se favorecen más con cada herramienta de Moodle.

Retomando las frecuencias de uso de cada herramienta (Gráfica 3) y observando su relación con las competencias genéricas que se consideran más favorecidas con cada herramienta (Gráfica 6), se observa lo siguiente: el trabajo en equipo se considera favorecido con las herramientas taller (37\%), foro (31\%) y base de datos (24\%). La responsabilidad se considera favorecida con las herramientas asistencia (59\%), tarea (50\%), lección (41\%), archivo (38\%), carpeta (34\%), cuestionario (33\%), página (32\%), URL (30\%), consulta, paquete SCORM (27\%) y HotPotatoes (25\%).

El liderazgo presenta pocas menciones, siendo la herramienta HotPotatoes la más aludida (13\% de los profesores). La cooperación se considera favorecida mediante la encuesta (25\%), el foro (24\%), el glosario y la base de datos (22\%). La comunicación se considera favorecida con el libro (38\%), el archivo (31\%), el cuestionario (28\%). La iniciativa se considera favorecida con la herramienta externa (28\%), HotPotatoes (24\%) y Paquete SCORM (22\%).

\subsection{Limitaciones.}

El caso de estudio descrito y los métodos usados para evaluar las herramientas podrían presentar ciertas limitaciones. Algunos aspectos que podrían afectar el resultado del estudio descrito son:

- El número de sujetos participantes. Posteriormente, se pretende repetir el experimento con un grupo mayor de participantes.

- El hecho de que no se consideran aspectos cuantitativos, como el tiempo invertido por cada sujeto en la realización de las tareas. Esto es parte de un trabajo futuro.

- El aspecto subjetivo de las respuestas de los participantes, debido a que están basadas sus percepciones acerca de la utilidad y facilidad de uso de cada herramienta.

- La veracidad de las respuestas proporcionadas. Aunque este aspecto fue controlado manteniendo el anonimato en el llenado de las encuestas, de esta forma se busca evitar que el participante coloque la respuesta que el experimentador desea obtener.

\section{Discusión}

En los resultados se observa que la muestra de docentes participantes proviene de diversas disciplinas y que el $84 \%$ de ellos utiliza alguna plataforma E-Learning, como Moodle, lo cual indica un buen punto de partida para el análisis de la información recabada. Al parecer, la institucionalización del MEFI en el año 2012 ha promovido la utilización de la plataforma Moodle pues el $48 \%$ de los docentes manifestó que, en el año 2015, llevaba menos de 3 años usando dicha plataforma.

En cuanto al uso de las herramientas disponibles en la plataforma Moodle, se corrobora la idea de ser escasa diversidad de herramientas que son utilizadas por los docentes universitarios. Se observa que las herramientas que los docentes consideran más favorecedoras de la evaluación son: tarea, cuestionario, encuesta y lección, en ese orden. Sin embargo, en cuanto a la usabilidad (facilidad de uso) de las mismas, se 
mencionan más: archivo, carpeta, URL y foro, en orden decreciente. Asimismo, por su frecuencia de uso destacan las herramientas archivo, tarea, carpeta, foro y URL, en ese orden. Esto indica que las herramientas más usadas coinciden con las de mayor usabilidad, pero contrastan con las que se consideran más favorecedoras de la evaluación del aprendizaje. Y aun cuando las funcionalidades pedagógicas de estas herramientas no sean totalmente claras, los usos que se les dan corresponden mayormente a la transferencia de información.

También se observa que los docentes suponen que las herramientas Tarea y Cuestionario pueden ser útiles para implementar la autoevaluación y la coevaluación, en tanto que no se concede mayor importancia a las herramientas Wiki y Blog, lo cual contrasta con los resultados encontrados en (Rodríguez Gómez y Dodero Beardo, 2008) con relación al Foro, Wiki y Blog, donde estos autores destacan la utilidad que éstas últimas tienen, especialmente para la evaluación por competencias.

Llama la atención que las herramientas consultas y asistencia aparecen como favorecedoras de la comunicación e interacción, a pesar de que en la Guía de Moodle para profesores (Seitzinger, 2010), no se considera que las consultas estén orientadas a estas finalidades, sino que resulta útil para recabar la opinión de los estudiantes sobre el curso o sobre algún tema. En el caso de la herramienta asistencia no se menciona en el material de Seitzinger, pues se incorporó en versiones más recientes de Moodle con el fin de registrar la asistencia de los estudiantes al salón de clases, permitiendo indicar que los estudiantes están presentes, tienen retardo, están ausentes, o bien, justificados (Moodle, 2017).

Analizando los altos porcentajes de profesores que no respondieron a la pregunta matricial sobre cuáles son las herramientas que más favorecen las competencias genéricas listadas (trabajo en equipo, responsabilidad, liderazgo, cooperación, comunicación escrita e iniciativa), se deduce que existe un amplio desconocimiento de las finalidades pedagógicas que dichas herramientas tienen. Entonces, pareciera que en cada curso se incluyen actividades para promover la adquisición de las competencias específicas correspondientes, pero las competencias genéricas no se están abordando en las aulas universitarias.

Analizando las relaciones expresadas entre las herramientas y las competencias favorecidas, se observa que la responsabilidad es una de las competencias que se puede abordar con más variedad de herramientas y, por el contrario, el liderazgo presenta menos posibilidades, al igual que la iniciativa y la cooperación. Asimismo, el alto porcentaje de docentes asocia la responsabilidad con las herramientas tarea y asistencia, lo cual indica que se tiene suficiente claridad de la relación existente entre ellas.

Al respecto, una de las medidas que ha implementado la UADY para fomentar el cambio hacia la evaluación por competencias consiste en la impartición de diplomados y talleres en los que se profundizan los conceptos y procedimientos relacionados con la evaluación de conocimientos, habilidades y actitudes, incluyendo cómo usar las herramientas disponibles en Moodle. Sin embargo, se sigue percibiendo poca claridad pues en la mayoría de los casos hace falta llevar la teoría a la práctica.

Análisis de la evaluación de competencias y su aplicación en un Sistema de Gestión del Aprendizaje. Cinhtia M. González-Segura, Michel García-García y Victor H. Menéndez-Domínguez. Página 16 de 20 
Otra actividad que se ha venido realizado en la UADY en los últimos años es un concurso de materiales de apoyo para la docencia, aunque todavía se percibe una mínima participación y los materiales presentados consisten principalmente en presentaciones de diapositivas, desaprovechando las ventajas inherentes a los objetos de aprendizaje, las aplicaciones móviles e interactivas, entre otros.

Para mejorar la situación observada se propone diseñar un modelo de evaluación de competencias que sirva de guía al docente durante el proceso de enseñanza-aprendizaje, en particular en el ámbito evaluativo; de tal forma que, a la par con el proceso metodológico, se cuente con un asistente de software que apoye su implementación, privilegiando la diversidad de estrategias de enseñanza y aprendizaje, con las que se aprovechen las ventajas presentes en la variedad de herramientas que se tienen disponibles actualmente para diversas finalidades pedagógicas, como se observa en la guía de herramientas para profesores (Seitzinger, 2010).

\section{Conclusiones}

En la primera parte del análisis realizado se ha presentado un listado de herramientas disponibles en un SGA con las funcionalidades asociadas a cada una de ellas, así como las principales finalidades pedagógicas que éstas tienen, basadas en la guía de herramientas para profesores (Seitzinger, 2010). Posteriormente, el estudio de caso implementado con 255 profesores universitarios cuyas disciplinas de especialidad son variadas, ha permitido lograr el objetivo planteado, analizando lo que ocurre en una universidad pública como la UADY respecto al uso de las herramientas que ofrece Moodle como plataforma institucional, para implementar el enfoque por competencias, en particular con el MEFI, que rige a esta institución.

Se lograron responder las preguntas planteadas al inicio, a partir de lo cual es posible concluir que las herramientas más usadas por los docentes son aquellas que presentan mayor facilidad de uso para ellos, a saber: archivo, tarea, carpeta, foro y URL. También se corroboró que son pocas las herramientas comúnmente usadas por los docentes, por lo que resulta conveniente promover un mayor uso de las mismas, o bien, ofrecer herramientas alternativas que satisfagan los requerimientos de las aulas.

Se observa también que existen ciertas discrepancias entre las finalidades pedagógicas declaradas por los desarrolladores de Moodle y los usos reales que los docentes hacen de las herramientas disponibles. Esto, junto con los resultados respecto a las herramientas que presentan mayor frecuencia y facilidad de uso, sugiere promover una mayor diversidad de tipos de actividades y herramientas, entre los docentes, de tal forma que se logre una evaluación de competencias más completa.

Como trabajo futuro se plantea implementar un modelo de evaluación acompañado de un asistente que guíe su uso, que en conjunto con los talleres de capacitación que se están impartiendo favorezcan la diversidad en el uso de las herramientas contenidas en Moodle, enfatizando los usos que benefician la función evaluadora del docente, bajo el enfoque por competencias. En dicho modelo se contempla incluir las modalidades de autoevaluación y coevaluación además de la heteroevaluación que típicamente se aplica en el aula. Se pretende que, con la guía del modelo y la ayuda del asistente, como complemento a los diplomados y talleres institucionales, el docente universitario pueda realizar su función evaluadora de manera más eficiente. 


\section{Agradecimientos}

A la Universidad Autónoma de Yucatán por las facilidades otorgadas para la obtención de los datos presentados en este documento, así como a los compañeros docentes que participaron.

Presentación del artículo: 10 de Octubre de 2017

Fecha de aprobación: 18 de febrero de 2018

Fecha de publicación: 31 de octubre de 2018

González-Segura, C.M., García-García, M., y Menéndez-Domínguez, V.H. (2018).

Análisis de la evaluación de competencias y su aplicación en un Sistema de Gestión del Aprendizaje. RED. Revista de Educación a Distancia, 58.

Consultado el (dd/mm/aaaa) en http://www.um.es/ead/red/58/

\section{Financiación}

Esta investigación no ha recibido ninguna subvención específica de los organismos de financiación en los sectores públicos, comerciales o sin fines de lucro.

\section{Referencias bibliográficas}

Cardona, S., Velez, J., \& Tobón, S. (2015). Modelo para la Evaluación de Competencias en Moodle. En Talento y Sociedad del Conocimiento. Cancún, Quintana Roo, México: Universidad de Quintana Roo. Recuperado de http://cifcomlatinoamerica.com/memorias/16\%20Sergio\%20cardona.pdf

De la Orden Oz, A. (2011). Reflexiones en torno a las competencias como objeto de evaluación en el ámbito educativo. Revista Electrónica de Investigación Educativa, 13(2), 1-21.

Esperón-Hernández, R. I., \& Ley Fuentes, M. (2005). Educación en línea en la UADY mediante un software libre. Apertura, 5(1), 67-72.

Frade, L. (2012). Los nudos existentes en la evaluación por competencias desde una visión del pensamiento complejo. En $5^{\circ}$ Congreso Nacional de Educación (pp. 21-39). Colima, México: SNTE.

González-Segura, C., Menéndez-Domínguez, V., Sánchez-Arias, V., \& Chan Nuñez, M. E. (2014). Propuesta de desarrollo y análisis de un módulo inteligente para gestionar/evaluar competencias universitarias desde Moodle. En XVI Encuentro Internacional Virtual Educa. Lima, Perú. Recuperado de http://www.virtualeduca.red/documentos/23/GonzalezCinhtia.pdf

Green, K. C. (2010). The 2010 national survey of information technology in U.S. higher education. En The Campus Computing Project. Recuperado de http://www.campuscomputing.net/sites/www.campuscomputing.net/files/GreenCampusComputing2010.pdf 
Gutiérrez-Castillo, J. J., Cabero-Almenara, J., \& Estrada-Vidal, L. I. (2017). Diseño y validación de un instrumento de evaluación de la competencia digital del estudiante universitario. Revista Espacios, 38(10), 1-27.

Ibarra Sáiz, M. S., Daniel Cabeza Sánchez, Álvaro R. León Rodríguez, Rodríguez Gómez, G., Gómez Ruiz, M. A., Gallego Noche, B., ... Cubero Ibáñez, J. (2010). EvalCOMIX en Moodle: Un medio para favorecer la participación de los estudiantes en la e-Evaluación. RED, Revista de Educación a Distancia, (Número especial dedicado a SPDECE 2010). Recuperado a partir de http://www.um.es/ead/red/24/Ibarra_Cabeza.pdf

Iglesias-Rodríguez, A., Olmos-Migueláñez, S., Torrecilla-Sánchez, E. M., \& Mena Marcos, J. J. (2014). Evaluar para optimizar el uso de la plataforma Moodle (studium) en el departamento de didáctica, organización y métodos de investigación. Tendencias pedagógicas, 23, 155-170.

Jiménez, V. (2013). Combinando las herramientas en Moodle para mejores resultados de aprendizaje. Presentado en Moodle Day, Ecuador. Recuperado de http://www.virtualepn.edu.ec/moodleday/descargar/presentacion_VJimenez.pdf

López, J. M., Romero, E., \& Ropero, E. (2010). Utilización de Moodle para el desarrollo y evaluación de competencias en los alumnos. Formación universitaria, 3, 45-52.

Menéndez-Domínguez, V. H., Castellanos-Bolaños, M. E., Cambranes Martínez, E., Medina Peralta, S., \& Zapata González, A. (2013). El proyecto AGORA, construyendo un Ecosistema para la Gestión de Objetos de Aprendizaje. En Tecnologías y Aprendizaje. Avances en Iberoamérica (Vol. 2, pp. 376-381). Cancún, Quintana Roo, México: Universidad Tecnológica de Cancún. Recuperado de http://puntodeencuentro.utn.edu.ar/wp-content/uploads/2016/10/ TecnologiasyAprendizaje.AvancesEnIberoamerica-Cun-CcIta-2013-Vol2.pdf

Moodle. (2017). Sitio web oficial de Moodle. Recuperado de https://moodle.net/stats/

Observatorio de innovación educativa. (2016, mayo). Evaluación del desempeño en el modelo educativo basado en competencias. Tecnológico de Monterrey. Recuperado de observatorio.itesm.mx

Palacios Burgos, F. J., \& Espinosa Martín, M. T. (2012). Módulo de evaluación de competencias para la plataforma educativa Moodle. En Sistemas y Tecnologías de la Información (pp. 117-120). Madrid, España. Recuperado de http://ieeexplore.ieee.org/xpl/mostRecentIssue.jsp?punumber=6255876

Rangel Baca, A. (2015). Competencias docentes digitales: propuesta de un perfil. Pixel-Bit. Revista de Medios y Educación, (46), 235-248.

Reyes Cabrera, W., Guillermo y Guillermo, C., Quiñones Pech, S., \& Zapata González, A. (2013). Diez años de educación en línea en una universidad pública: el caso de la facultad de educación de la UADY. XXI Encuentro Internacional de Educación a Distancia.

Rodríguez Gómez, G., \& Dodero Beardo, J. M. (2008). Evaluación de competencias con Herramientas de Interacción Dialógica Asíncronas. En EvalHIDA: Evaluación de competencias con herramientas de interacción dialógica asíncronas (foros, blogs y wikis). España: Servicio de Publicaciones de la Universidad de Cádiz. 
Sanchez Ambriz, M. L., \& Martínez Balboa, S. (2010). Una experiencia para evaluar competencias a través de Moodle. Revista Cognición 27, 6(7), 1-13.

Sancho-Vinuesa, T., \& Escudero-Viladoms, N. (2012). A Proposal for Formative Assessment with Automatic Feedback on an Online Mathematics Subject. RUSC. Universities and Knowledge Society Journal; Vol 9, No 2 (July 2012), 9(2), 59-79.

Seitzinger, J. (2010). Moodle tool guide for teachers. Recuperado de http://www.catspyjamas.net/wp-content/uploads/2010/05/MoodleToolGuideforTeachers_May2010_JS .pdf

Tobón, S., Pimienta, J., \& García, J. (2010). Secuencias didácticas: aprendizaje y evaluación de competencias. México: PEARSON EDUCACIÓN.

UADY. (2012). Modelo Educativo para la Formación Integral. Universidad Autónoma de Yucatán.

UADY. (2013). Programa Institucional de Habilitación en el MEFI. Universidad Autónoma de Yucatán. Recuperado de http://www.diie.dgda.uady.mx/new.php?id=27\#

UADY. (2014). UADY Global. Recuperado de http://www.slideshare.net/uadyglobal/universidad-autnoma-de-yucatn

UADY. (2017). Plan de Desarrollo Institucional 2014-2022. Universidad Autónoma de Yucatán. Recuperado de http://www.pdi.uady.mx/cuarta_agenda/doc/Accion\%207.pdf

Urbina Nájara, A. B., Medina Nieto, M. A., \& Vargas, G. (2013). Uso de Moodle para evaluar competencias cognitivas en ciencias exactas. Educere, 17(56), 51-58.

Valverde Berrocoso, J., Revuelta Domínguez, F. I., \& Fernández Sánchez, M. R. (2012). Modelos de evaluación por competencias a través de un sistema de gestión de aprendizaje. Experiencias en la formación inicial del profesorado. Revista Iberoamericana de Educación, (60), 51-62.

Zabala, A., \& Arnau, L. (2007). IDEA CLAVE 11. Evaluar competencias es evaluar procesos en la resolución de situaciones problema. En 11 ideas clave: cómo aprender y enseñar competencias. México: Graó/Colofón.

Zapata, M. (2010). Estrategias de evaluación de competencias en entornos virtuales de aprendizaje. RED, Revista de Educación a Distancia, (1). Recuperado de http://www.um.es/ead/reddusc/1 